\title{
Low-Energy Electron Elastic Total Cross Sections for the Large Actinide Atoms Cf, Fm and Md
}

\author{
Zineb Felfli ${ }^{1} \&$ Alfred Z. Msezane ${ }^{1}$ \\ ${ }^{1}$ Department of Physics and Center for Theoretical Studies of Physical Systems, Clark Atlanta University, Atlanta, \\ USA
}

Correspondence: Alfred Z. Msezane, Department of Physics and Center for Theoretical Studies of Physical Systems, Clark Atlanta University, Atlanta, Georgia 30314, USA.

Received: January 11, 2022

Accepted: February 10, 2022

Online Published: March 1, 2022

doi:10.5539/apr.v14n1p15

URL: https://doi.org/10.5539/apr.v14n1p15

\begin{abstract}
The rigorous Regge pole method has been used to investigate negative-ion formation in the large actinide atoms $\mathrm{Cf}, \mathrm{Fm}$ and Md through the elastic total cross sections (TCSs) calculation in the electron impact energy range 0.0 $\leq \mathrm{E} \leq 10.0 \mathrm{eV}$. Ground, metastable and excited negative-ion formation as well as shape resonances (SRs) and Ramsauer-Townsend (R-T) minima are found to characterize generally the TCSs which also exhibit fullerene molecular behavior near threshold through the TCSs of the highest excited states, while maintaining atomic character through the ground state TCSs. Additionally, a polarization-induced metastable TCS with a pronounced SR appears for the first time very close to threshold in the Cf TCSs, having flipped over from a deep R-T minimum near threshold in the Bk TCSs. This behavior manifests the impact of the size effect and the 6d-orbital collapse as well as demonstrates the sensitivity of the R-T minima and the SRs to the electronic structure of these atoms, thereby permitting their first ever use as novel confirmation of $\mathrm{Cf}$ as a transitional element in the actinide series. Rigorous and unambiguous ground, metastable and excited anionic states BEs extracted from the TCSs are compared with the existing electron affinities
\end{abstract}

PACs.: $34.80 . \mathrm{Bm}$

Keywords: generalized bound states, electron correlations, anionic binding energies, Regge poles, actinide atoms, elastic cross sections

\section{Introduction}

The radioactive actinide atoms, including the At atom are challenging to handle experimentally; therefore making the production of reliable theoretical data essential. These atoms, vital in nuclear power plants, energy production and medicine to name a few are important in defense operations and nuclear weapons. In particular, $\mathrm{Pu}$ is used in nuclear reactors and in nuclear bombs as well. Until very recently, there were no measured electron affinities (EAs) of the actinide atoms. A great achievement was the recent first time ever measurement of the EA of the highly radioactive At atom (Leimbach et al., 2020) as well as of the actinide atoms Th (Tang, et al., 2019) and U (Tang, Lu, Liu \& Ning, 2021; Ciborowski et al., 2021). For the At atom the MCDHF-calculated value (Si \& Froese Fischer, 2018) agrees excellently with the measured EA (Leimbach et al., 2020). Leimbach et al., 2020 employed Coupled-Cluster method. Furthermore, in ( $\mathrm{Si} \&$ Froese Fischer, 2018) the EAs from various sophisticated theoretical methods, including of (Li et al., 2012) were contrasted. For the atoms At (Leimbach et al., 2020), Au (Hotop \& Lineberger, 2003; Andersen, Haugen, \& Hotop, 1999; Zheng et al., 2007) and Pt(Hotop \& Lineberger, 2003; Gibson, Davies, \& Larson, 1993; Bilodeau, et al., 1999) as well as the fullerene molecules $\mathrm{C}_{20}$ through $\mathrm{C}_{92}$ (Msezane \& Felfli, 2018a; Felfli \& Msezane, 2018a) the measured EAs generally matched excellently the Regge pole-calculated BEs of the ground states of the formed negative ions during the collisions. This gives great credence to our interpretation of the EAs of these heavy multi-electron systems, viz. as corresponding to the ground states BEs of the formed negative ions during the collisions. Indeed, the Regge pole method requires no assistance whatsoever from either experiment or other theory to achieve the remarkable feat.

The Regge pole-calculated low-energy electron elastic scattering total cross sections (TCSs) and the attendant negative-ion binding energies (BEs) of the formed negative ions during the electron collisions with the Cf, Fm and $\mathrm{Md}$ atoms are presented in this paper to complete the data for the actinide atoms published in (Felfli \& 
Msezane, 2019; Msezane \& Felfli, 2021). These TCSs are important for the determination and elucidation of the anionic BEs of the formed anions during the electron collision with multi-electron atoms (Felfli \& Msezane, 2019; Msezane \& Felfli, 2021; Felfli \& Msezane, 2020; Felfli \& Msezane, 2018b) and fullerene molecules (Msezane \& Felfli, 2018a; Felfli \& Msezane, 2018a). The energy positions of the dramatically sharp resonances in the TCSs correspond to the anion BEs of the formed negative ions during the electron collision with the ground, metastable and excited states atoms, see for example Table 1 of (Msezane \& Felfli, 2021). These negative-ion BEs yield the challenging to calculate, using conventional theoretical methods, EAs of multi-electron atoms such as the actinide and lanthanide atoms (Msezane \& Felfli, 2021; Felfli \& Msezane, 2020; Felfli \& Msezane, 2018b) and fullerene molecules (Msezane \& Felfli, 2018a; Felfli \& Msezane, 2018a).

In the recent measurement of the EAs of the actinide atoms Th (Tang, et al., 2019) and U (Tang, Lu, Liu \& Ning, 2021; Ciborowski, et al., 2021) the experimentalists claimed that their EAs corresponded to the BEs of the weakly bound electron to the neutral atoms. Importantly, the measured EAs of Th and U are close to the Regge pole-calculated BEs of their excited anionic states and not to their ground states (Msezane \& Felfli, 2021). Clearly, there are two prevailing viewpoints regarding the interpretation of the measured EA: 1) The first considers the EA to correspond to the electron $\mathrm{BE}$ in the ground state of the formed anion during the collision; and 2) The second view identifies the measured EA with the BE of the attached electron in an excited state of the formed anion. These two viewpoints need to be reconciled to avoid the ambiguous and confusing meaning of the EAs of complex multi-electron atoms now populating the literature, see for example ( Felfli \& Msezane, 2020; Felfli \& Msezane, 2018b).

Multi-electron systems such as the lanthanide and actinide atoms consist of large numbers of electrons and have open $\mathrm{d}$ - and f-subshell electrons. The resultant large intricate and diverse electron configurations present formidable computational complexities when conventional theoretical methods are used to calculate the EAs of multi-electron systems. Indeed, many existing experimental and sophisticated theoretical EAs of the actinides and lanthanides are difficult to interpret (Msezane \& Felfli, 2021; Felfli \& Msezane, 2020; Felfli \& Msezane, 2018b; Msezane \& Felfli, 2018b). Thus a rigorous theoretical method such as the Regge pole method is appropriate for calculating unambiguous and reliable EAs. In the Regge pole method the EAs of multi-electron systems correspond to electron BEs in the ground, metastable or excited state of the formed negative ions during the collision process. Regardless of the interpretation adopted for the EA, our data will be applicable for use in guiding measurements.

Most existing theoretical methods used for calculating the anionic BEs of complex multi-electron systems (atoms and fullerene molecules) are structure-based. Therefore, the results obtained through these methods such as the EAs of multi-electron systems are often riddled with uncertainties and lack definitiveness. The general weakness of these methods lies in that they employ large configuration-interaction expansions demanded by the diffuseness of the wave functions for negative ions. The theoretical methods developed in atomic and molecular physics, were designed with the primordial task of reproducing experimental results with high accuracies. Indeed, this effort resulted in the development of very sophisticated and powerful methods for the most part, that provided agreement with experiment, but did very little in unravelling the intricate details of the important underlying physics and providing physical insights into the essential physical effects important to a particular process; e.g. electron affinity calculation of heavy multi-electron atoms and fullerene molecules. Naturally, these theoretical methods lack the predictive power to guide measurements.

The conventional quantum mechanical approach to scattering problems expresses the desired solution as a partial-wave series where the summation is over the orbital (or total) angular momentum quantum number. The partial-wave series is notoriously slowly convergent, particularly when the wavelength of the incoming particle is much smaller than the range of the scattering potential. The partial-wave expansion may contain hundreds, if not thousands, of terms. However, if the angular momentum is allowed to become complex-valued, this slow convergence can be overcome, viz. by replacing the slowly convergent partial-wave series with a more rapidly converging series. This leads us to the idea of Regge poles. Simply put, Regge poles are generalized bound-states, i.e. solutions of the Schrödinger equation where the energy $\mathrm{E}$ is real, positive and the angular momentum $\lambda$ is complex. The complex angular momentum (CAM) methods have the advantage in that the calculations are based on a rigorous definition of resonances, viz. as singularities of the S-matrix, see also (Sokolovski, et al., 2007a; Connor, 1990).

It has now been established firmly that the low-energy electron elastic scattering TCSs for complex multi-electron systems calculated using the rigorous Regge pole method are characterized generally by ground, metastable and excited states negative-ion formation, Ramsauer-Townsend (R-T) minima and shape resonances (SRs), see for example (Msezane \& Felfli, 2018a; Felfli \& Msezane, 2019; Msezane \& Felfli, 2021; Felfli \& 
Msezane, 2020; Felfli \& Msezane, 2018b). The extracted anionic BEs from the TCSs yield the unambiguous and definitive EAs. And the EAs provide a stringent test of theoretical methods when the calculated EAs are compared with those from reliable measurements.

The paper is organized as follows: Sections 2 and 3 present the brief Method of Calculation and the Results, respectively. Section 4 deals with the Summary and Conclusion.

\section{Method of Calculation}

Briefly, the calculation of the electron elastic TCSs yielding the anionic BEs uses the rigorous Regge pole method. Regge poles, singularities of the S-matrix, rigorously define resonances (Frautschi, 1963; D' Alfaro \& Regge, 1965). In the physical sheets of the complex plane the Regge poles correspond to bound states (Omnes \& Froissart, 1963). Confirmed in (Hiscox, Brown, \& Marletta, 2004), Regge poles formed during low-energy electron elastic scattering become stable bound states. In the Regge pole, also known as the CAM method the important and revealing energy-dependent Regge trajectories are also calculated. Their effective use in low-energy electron scattering has been demonstrated in for example (Felfli, Msezane, \& Sokolovski, 2009; Thylwe, 2012).

\subsection{Elastic Scattering Total Cross Section}

The near-threshold electron-atom/fullerene scattering TCS resulting in negative ion formation as resonances is calculated using the Mulholland formula (Mulholland, 1928). In the form below, the TCS fully embeds the essential electron-electron correlation effects (Macek, Krstic, \& Ovchinnikov, 2004; Sokolovski, et al., 2007b) (atomic units are used throughout):

$$
\begin{aligned}
& \sigma_{t o t}(E)=4 \pi k^{-2} \int_{0}^{\infty} \operatorname{Re}[1-S(\lambda)] \lambda d \lambda \\
& -8 \pi^{2} k^{-2} \sum_{n} \operatorname{Im} \frac{\lambda_{n} \rho_{n}}{1+\exp \left(-2 \pi i \lambda_{n}\right)}+I(E)
\end{aligned}
$$

In Eq. (1) $\mathrm{S}(\lambda)$ is the S-matrix, $k=\sqrt{2 m E}, m$ being the mass and $E$ the impact energy, $\rho_{n}$ is the residue of the S-matrix at the $n^{\text {th }}$ pole, $\lambda_{n}$ and $I(E)$ contains the contributions from the integrals along the imaginary $\lambda$-axis ( $\lambda$ is the complex angular momentum); its contribution has been demonstrated to be negligible (Felfli, Msezane, $\&$ Sokolovski, 2009). The importance of $\operatorname{Im} \lambda_{n}(\mathrm{E})$ is carefully explained in (Msezane \& Felfli, 2018b). Inter alia, it determines the extent of the resonance contribution to the TCS, arising from the "creeping wave" (Sokolovski, et al., 2007a) which, after encircling the core many times, may return to decay in the forward direction. Indeed, if the wave returns to the forward direction several times to accumulate the phase, and $\operatorname{Im} \lambda_{n}(\mathrm{E}) \ll<$, the resonance in (1) will contribute to the TCS. However, $\operatorname{Im} \lambda_{n}(\mathrm{E})$ could not be too small since if $\operatorname{Im} \lambda_{n}(\mathrm{E}) \rightarrow 0$ the resonance becomes a bound state (the angular life, $1 /(\operatorname{Im} \lambda(\mathrm{E})) \rightarrow \infty$ (Sokolovski, et al., 2007a; Conner, 1990) implying that the system can never decay) which is inaccessible to the incident electron since then $\mathrm{E}$ must be negative. Consequently, in this limit the residue $\rho_{n}$ would vanish as well.

\subsection{The Potential}

Here we consider the incident electron to interact with the complex heavy multi-electron system (atoms and fullerene molecules) without consideration of the complicated details of the electronic structure of the system itself as was done in (Dolmatov, Amusia, \& Chernysheva, 2017). Therefore, within the Thomas-Fermi theory, Felfli et al. 2004 generated the robust Avdonina-Belov-Felfli (ABF) potential which embeds the vital core-polarization interaction

$$
U(r)=-\frac{Z}{r\left(1+\alpha Z^{1 / 3} r\right)\left(1+\beta Z^{2 / 3} r^{2}\right)}
$$

In Eq. (2) $Z$ is the nuclear charge, $\alpha$ and $\beta$ are variation parameters. For small $r$, the potential describes Coulomb attraction between an electron and a nucleus, $\mathrm{U}(r) \sim-\mathrm{Z} / r$, while at large distances it has the appropriate asymptotic behavior, viz. $\sim-1 /\left(\alpha \beta \mathrm{r}^{4}\right)$ and accounts properly for the polarization interaction at low energies. For an electron, the source of the bound states giving rise to Regge trajectories is the attractive Coulomb well it 
experiences near the nucleus. The addition of the centrifugal term to the well 'squeezes' these states into the continuum (Sokolovski, et al., 2007a).

The strength of this extensively studied potential (Belov, et al., 2010; Belov, et al., 2004; Thylwe \& McCabe, 2014) lies in that it has five turning points and four poles connected by four cuts in the complex plane. The presence of the powers of $\mathrm{Z}$ as coefficients of $r$ and $r^{2}$ in Eq. (2) ensures that spherical and non-spherical atoms and fullerenes are correctly treated. Also appropriately treated are small and large systems. The effective potential $V(r)=U(r)+\lambda(\lambda+1) / 2 r^{2}$ is considered here as a continuous function of the variables $r$ and complex $\lambda$. The details of the numerical evaluation of the TCSs have been described in (Sokolovski, et al., 2007b) see also (Felfli, Msezane, \& Sokolovski, 2009). We calculate the S-matrix, $S(\lambda, k)$ poles positions and residues of the Schrödinger equation following a method similar to that of Burke and Tate (Burke \& Tate, 1969). In the method the two linearly independent solutions of the Schrödinger equation are evaluated as Bessel functions of complex order and the S-matrix, which is defined by the asymptotic boundary condition of the solution of the Schrödinger equation, is thus evaluated. Further details of the calculation may be found in (Burke \& Tate, 1969). We emphasize here that the investigation of the Regge trajectories, $\operatorname{Im} \lambda_{n}(\mathrm{E})$ versus $\operatorname{Re} \lambda_{n}(\mathrm{E})$ allows us to readily identify the electron impact energy range where Relativity is unimportant in the calculation of the TCSs, i.e. at small electron impact energies. Also, the use of the $\operatorname{Im} \lambda_{n}(\mathrm{E})$ allows us to differentiate among the important ground, metastable and excited negative-ion formation as well as the shape resonances, see also (Felfli, Msezane, \& Sokolovski, 2009). The very small value of $\operatorname{Im} \lambda_{n}(\mathrm{E})$ indicates the ground state negative-ion formation.

\section{Results}

Previously, we investigated low-energy electron elastic TCSs using the rigorous Regge pole method and extracted the anionic BEs of the actinide atoms Th through Pa (Felfli \& Msezane, 2019) and Am through Lr (Msezane \& Felfli, 2021). And the anionic BEs were compared with the available calculated (O’Malley \& Beck, 2009; Guo \& Whitehead, 1989; Eliav, Kaldor, \& Ishikawa, 1995; Borschevsky, et al., 2007) and the measured (Tang, et al., 2019; Tang, Lu, Liu \& Ning, 2021; Ciborowski, et al., 2021) EAs. However, the TCSs for the large actinide atoms Cf, Fm and Md were never calculated. The importance of the present data when taken together with those of (Felfli \& Msezane, 2019; Msezane \& Felfli, 2021) is in the demonstration that the near threshold R-T minimum in the Bk TCSs flips over for the first time to a SR in the Cf TCSs rather than in the Es TCSs as was conveyed in (Msezane \& Felfli, 2021). Generally, the TCSs for these atoms, presented for the first time in Figures 1, 2 and 3, resemble those of the large actinide atoms already presented in (Msezane \& Felfli, 2021). At a glance they appear a little complicated to analyze and understand. However, since each Figure consists of the ground, metastable and excited states TCSs they can be readily analyzed individually and understood.

\subsection{Ground State Total Cross Sections}

We first focus on the TCSs of Figure 1, in particular the ground state TCS (red curve). From threshold this TCS decreases monotonically, with the increase of the electron energy, E. The TCS reaches the first R-T minimum at about $1.12 \mathrm{eV}$. As E increases the electron becomes trapped by the centrifugal potential as manifested by the appearance of the SR at about $1.81 \mathrm{eV}$. A further increase in energy, causes the electron to leak out of the barrier, reaching the absolute R-T minimum at $3.34 \mathrm{eV}$, where the electron becomes attached to the atom forming a long-lived negative ion with the $\mathrm{BE}$ value of $3.32 \mathrm{eV}$. For ground state collisions the $\operatorname{Im} \lambda_{n}(\mathrm{E})$ is very small, see (Felfli, Msezane, \& Sokolovski, 2009); its inverse determines the angular life of the formed negative ion $\mathrm{Cf}^{-}$. It is noted here that the $\operatorname{Im} \lambda_{n}(\mathrm{E})$ cannot be too small because a true bound state would require that $\mathrm{E}$ be less than zero (Sokolovski, et al., 2007a).

\subsection{Metastable States Total Cross Sections}

The TCSs of Figure 1 have two metastable TCSs, represented by the blue and the orange curves. The blue curve, contrary to the behavior of the ground state TCS curve, increases from threshold with increasing E. Then the electron becomes trapped by the centrifugal barrier at about $0.042 \mathrm{eV}$, representing the position of the SR. With further increase of the E an R-T minimum is reached at about $0.576 \mathrm{eV}$, representing the first maximum polarization before the electron becomes trapped by the centrifugal potential at $0.80 \mathrm{eV}$. As the energy increases, the electron leaks out of the potential barrier, and the atom reaches maximum polarization at about $1.71 \mathrm{eV}$ where the electron becomes bound to the atom forming a negative ion with the $\mathrm{BE}$ value of $1.70 \mathrm{eV}$. 
The second metastable state TCS is represented by the orange curve. At the electron energy of $0.01 \mathrm{eV}$, this TCS is increasing with the increasing impact energy, reaching a maximum value of about $1.03 \times 10^{4}$ a.u. at $0.022 \mathrm{eV}$ where the electron is trapped by the centrifugal potential, manifested through the SR. With the further increase of $\mathrm{E}$ the TCS decreases precipitously by several orders-of-magnitude before reaching maximum polarization at the first $\mathrm{R}-\mathrm{T}$ minimum at $0.281 \mathrm{eV}$. As the $\mathrm{E}$ increases, the electron becomes trapped by the centrifugal barrier at $0.381 \mathrm{eV}$, shown by the SR. With slight increase of the electron energy, the TCS drops significantly, reaching the second R-T minimum at $0.956 \mathrm{eV}$. It is noted that this second metastable TCS, characterized by the SR very near threshold previously appeared with a deep R-T minimum in the TCSs of the smaller actinide atom Pu (Felfli \& Msezane, 2019). It first flipped over from an R-T minimum near the threshold to a SR in the TCSs of the Es atom (Msezane \& Felfli, 2021). This flip was attributed to the increase in the size of the actinide atom and the second 6d-orbital collapse in transitioning from $\mathrm{Cm}[\mathrm{Rn}] 7 \mathrm{~s}^{2} 5 \mathrm{f}^{7} 6 \mathrm{~d}$ to $\mathrm{Bk}[\mathrm{Rn}] 7 \mathrm{~s}^{2} 5 \mathrm{f}^{9}$, impacting significantly the polarization interaction. This provides a new mechanism of tuning R-T minima and SRs through the polarization interaction.

\subsection{Excited States Total Cross Sections}

There are two excited states TCSs in Figure 1; they are represented by the brown and the green curves. They are viewed appropriately as the lowest and the highest excited states TCSs, respectively.

The lowest excited state TCS (brown curve) decreases rapidly from threshold with the increase of the electron energy from $0.01 \mathrm{eV}$. It reaches the deep R-T minimum at about $0.083 \mathrm{eV}$, manifesting maximum polarization of the Cf atom at this energy. Beyond this R-T minimum, the TCS increases significantly, reaching its first maximum at $0.241 \mathrm{eV}$, represented by the SR before decreasing. Soon after leaking out of the potential barrier, the electron reaches the second R-T minimum where it becomes attached to the atom forming a stable negative ion with the $\mathrm{BE}$ value of $0.577 \mathrm{eV}$.

The highest excited state TCS (green curve) behaves similarly to the lowest excited state TCS as the electron energy increases from near threshold to about $0.8 \mathrm{eV}$. However, the positions of the R-T minima, the SR and the dramatically sharp resonance at $0.272 \mathrm{eV}$ all have been pulled closer to the threshold relative to the second excited state values, manifesting that this excited state TCS corresponds to a weakly interacting electron with the $\mathrm{Cf}$ atom. The TCS goes through the first R-T minimum at $0.049 \mathrm{eV}$ before reaching the SR at $0.142 \mathrm{eV}$. It then decreases rapidly, reaching the second R-T minimum at about $0.272 \mathrm{eV}$ where the electron becomes attached to the atom, forming the stable negative ion with the BE value of $0.272 \mathrm{eV}$. Indeed, the ratio R of the TCS at the second R-T minimum to that at the first R-T minimum is less than unity (0.88), manifesting fullerene molecular behavior (Msezane \& Felfli, 2018a).

Before we continue with the discussion of Figures 2 and 3, the following remarks are appropriate here concerning the TCSs of atomic Cf. They are also applicable to the results of Figs. 2 and 3. 1) The appearance of the characteristic R-T minima in the TCSs of Figure 1 manifests that the crucial polarization interaction has been accounted for adequately in our calculation, consistent with the conclusion by (Johnson \& Guet, 1994). These R-T minima were also observed in the low-energy electron elastic scattering cross sections of the heavy alkali-metal atoms Rb, Cs and Fr calculated using the Dirac R-matrix (Bahrim \& Thumm, 2000). They are important in understanding sympathetic cooling and the production of cold molecules from natural fermions (Aubin, et al., 2006). 2) The dominant polarization-induced metastable TCS is characterized by a pronounced SR very close to threshold. This TCS exhibiting a deep R-T minimum near threshold first appeared in the TCSs of the smaller actinide atom Pu (Felfli \& Msezane, 2019); the R-T minimum has flipped over to a SR in the Cf TCSs. We attribute these novel manifestations to size effects and 6d-orbital collapse impacting significantly the polarization interaction. This provides a new mechanism of tuning SRs and R-T minima through the polarization interaction.

Notably, close to the excited and metastable states anionic BEs, represented by the dramatically sharp resonances are SRs. Therefore, the delineation and identification of the dramatically sharp resonances are very important, particularly for those experimenters defining the EA as corresponding to the anionic BE of the least bound electron to the neutral atom (Tang, et al., 2019; Tang, Lu, Liu \& Ning, 2021; Ciborowski, et al., 2021). However, if the EA is identified with the $\mathrm{BE}$ of the anionic ground state of the formed negative ion as in the cases of the Au, Pt and At atoms, there will be no ambiguity. The data for the $\mathrm{Cf}$ atom are summarized in Table 1 where they are compared with those for the Fm and Md atoms. Their anionic BEs are compared with the available EAs; there are no measured EAs of the investigated atoms to our knowledge. 


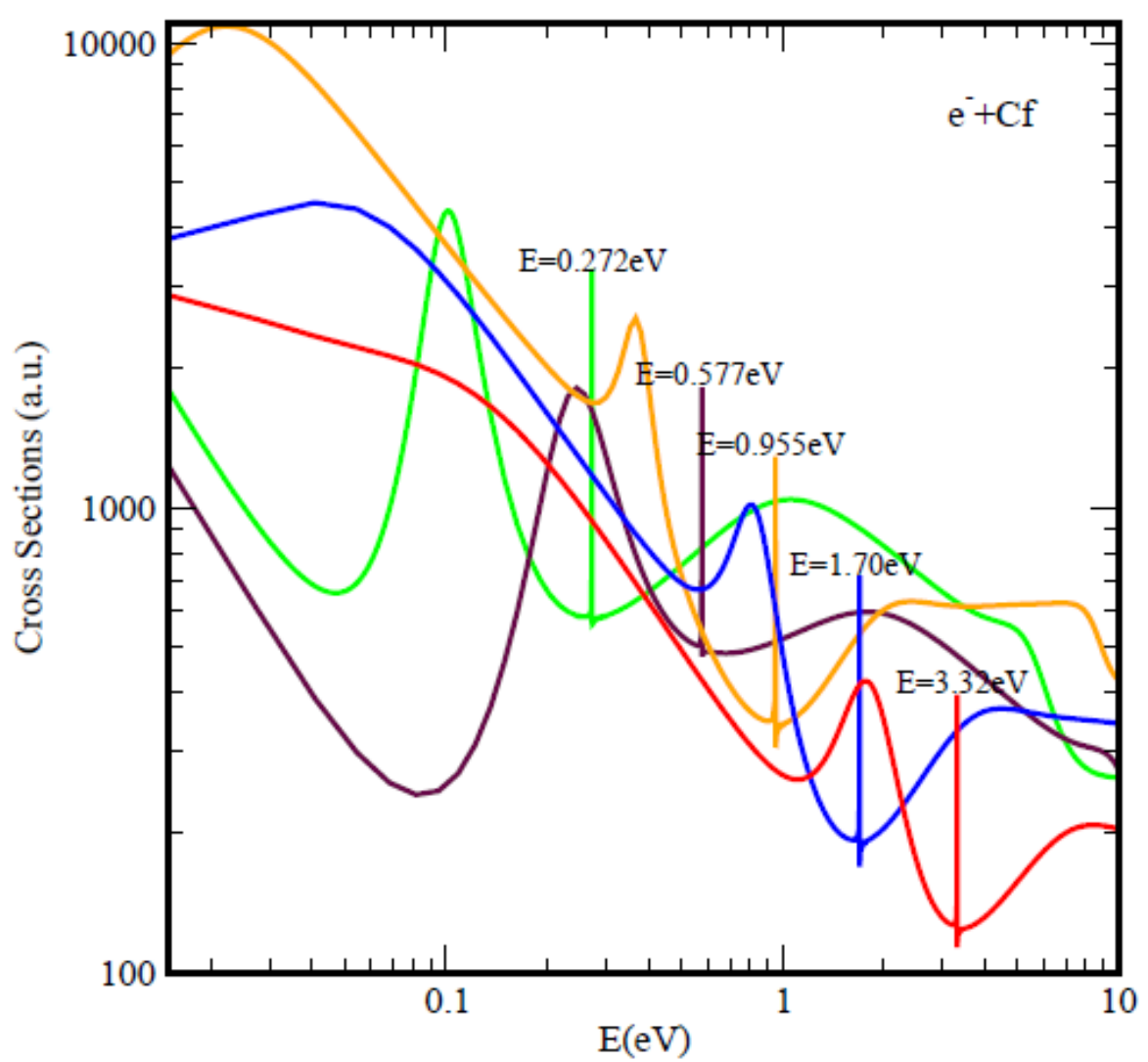

Figure 1. Total cross sections (a.u) for electron-Cf scattering. Red, blue and orange curves represent, ground and metastable TCSs, respectively. Brown and green curves are two excited states TCSs. The polarization-induced TCS due to size effect is the orange curve.

The TCSs in Figures 2 and 3 are for the Fm and Md atoms, respectively. They can be analyzed similarly to those for the $\mathrm{Cf}$ atom. Since we are interested in the dramatically sharp resonances, representing the formed negative ions during the collisions with the anionic BEs; these are indicated in the Figures for the ground, metastable and excited states TCSs. These BEs are summarized in Table 1 as well. Although the TCSs of the three atoms Cf, Fm and $\mathrm{Md}$ resemble each other, there are notable differences. For example, their ground states anionic BEs are $3.32 \mathrm{eV}, 3.47 \mathrm{eV}$ and $3.77 \mathrm{eV}$, respectively, while their highest excited states $\mathrm{BEs}$ are $0.272 \mathrm{eV}, 0.268 \mathrm{eV}$ and $0.259 \mathrm{eV}$, respectively. Note the reversed magnitudes of the excited states compared to those of the ground states. As pointed out, whether the EA is defined to correspond to the BE of electron attachment in the ground or excited state of the formed anion, our results are equally applicable. 


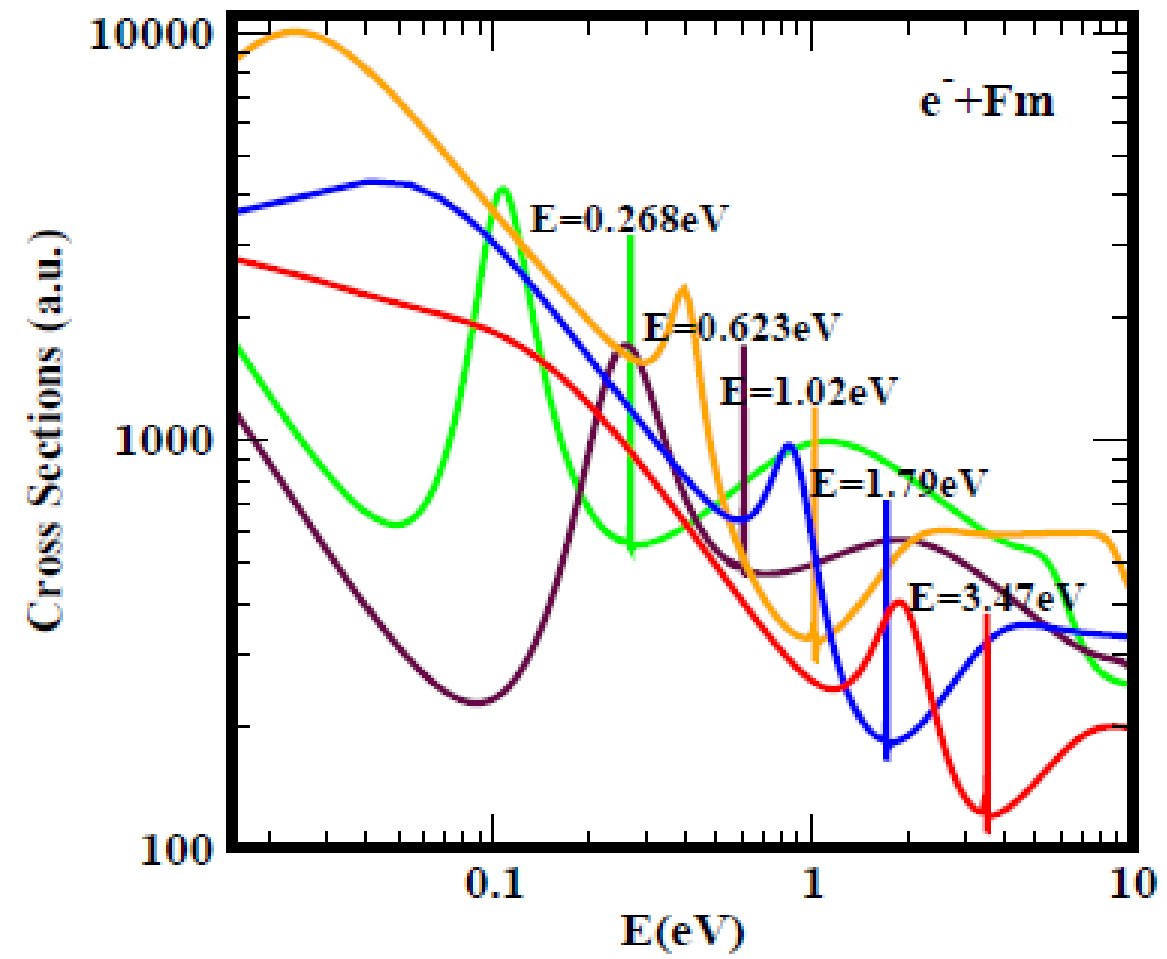

Figure 2. Total cross sections (a.u) for electron-Fm scattering. Red, blue and orange curves represent, ground and metastable TCSs, respectively. Brown and green curves are two excited states TCSs. The polarization-induced TCS due to size effect is the orange curve.

In Table 1 is presented the Regge pole-calculated anionic BEs of the ground (GRS), metastable (MS-1; MS-2) and excited (EX-1; EX-2) states of the investigated atoms as well as the ground state (GRS) R-T minima and the ratio $\mathrm{R}$. The anionic BEs of the formed negative ions in the ground, metastable and excited states are compared with the BEs and the EAs of (O'Malley \& Beck, 2009; Guo \& Whitehead, 1989) calculated using the Relativistic configuration-interaction (RCI) method and the local spin-density-functional theory, respectively. The values of (O'Malley \& Beck, 2009) are available for only the Cf atom and are significantly smaller than our BEs. The EAs of (Guo \& Whitehead, 1989) are riddled with uncertainties and consist of positive and negative EA values. These demonstrate the difficulties experienced by sophisticated theoretical methods in producing unambiguous and reliable $\mathrm{BEs} / \mathrm{EAs}$ of the $\mathrm{Cf}, \mathrm{Fm}$ and $\mathrm{Md}$ actinide atoms and point out to the need for reliable theoretical BEs/EAs values to guide measurements.

The EAs of Fm and Md of (Guo \& Whitehead, 1989) in Table 1 are of interest when contrasted with our anionic BEs. For Fm the top value $(0.597 \mathrm{eV})$ is close to our BE of the second excited state $(0.623 \mathrm{eV})$ while the bottom EA $(0.354 \mathrm{eV})$ is close to our BE of the highest excited state $(0.268 \mathrm{eV})$. However, for Md the EA value $(1.224 \mathrm{eV})$ compares with our $\mathrm{BE}$ of the second metastable state $(0.996 \mathrm{eV})$ while the bottom value $(0.978 \mathrm{eV})$ is close to our $\mathrm{BE}$ of the second excited state $(0.700 \mathrm{eV})$. Importantly, we can conclude that for the Fm atom the EAs of (Guo \& Whitehead, 1989) correspond to our BEs of excited states. However, for Md the first EA value of (Guo \& Whitehead, 1989) corresponds to our metastable BE, while the second EA value is identifiable with our BE of an excited state. Indeed, the electronic structures of these actinides are very complicated to even expect agreement of any kind. And, Table 1 demonstrates the need for experimental EAs as well as reliable and unambiguous theoretical EAs; the available EAs are riddled with uncertainties.

Finally, we see from the R ratio, less than unity, that the TCSs of the highest excited states of the Cf, Fm and Md atoms exhibit fullerene molecular behavior (Msezane \& Felfli, 2018a) while the atomic character is maintained through the ground states TCSs. 


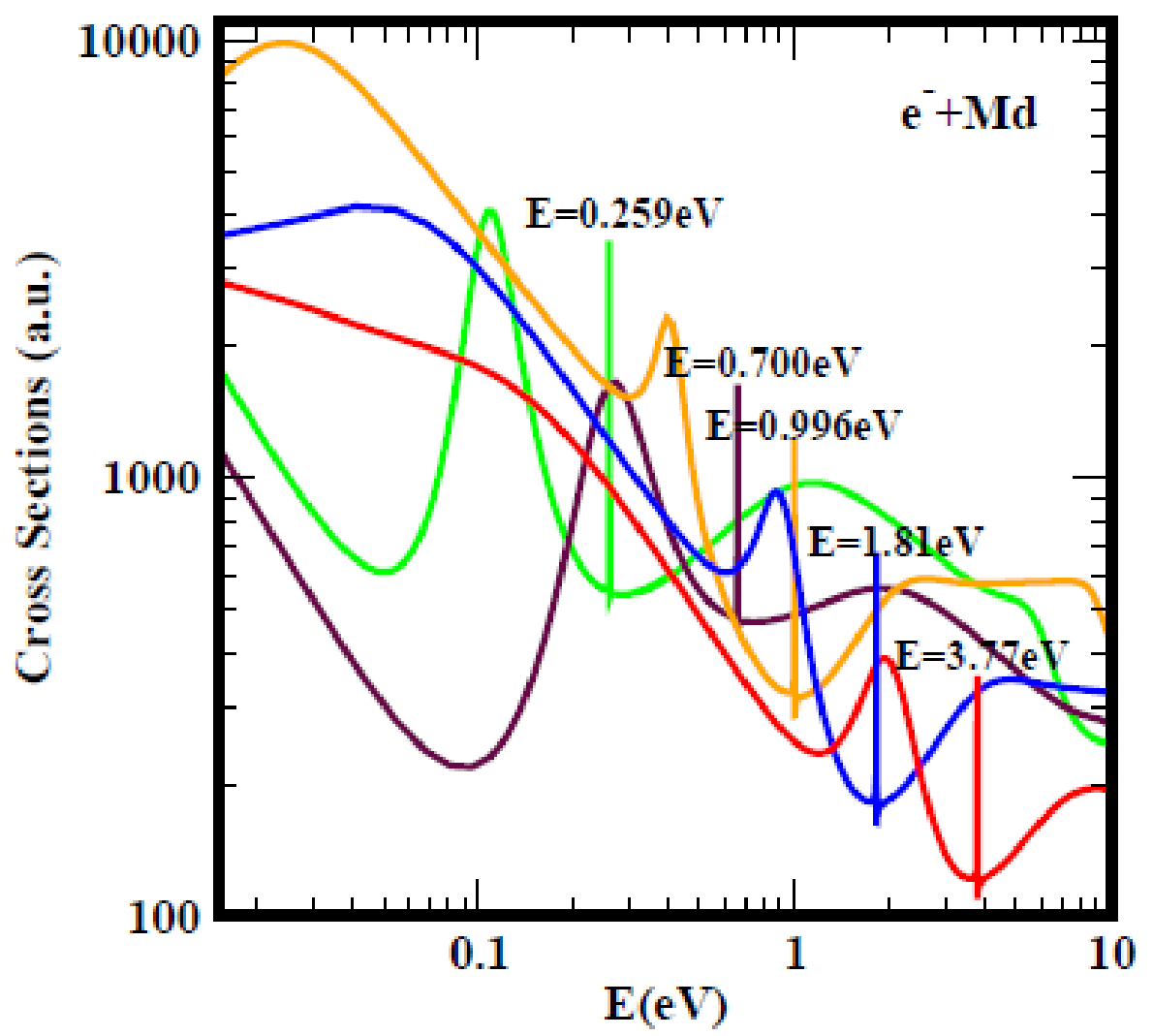

Figure 3. Total cross sections (a.u) for electron-Md scattering. Red, blue and orange curves represent, ground and metastable TCSs, respectively. Brown and green curves are two excited states TCSs. The polarization-induced TCS due to size effect is the orange curve.

Table 1. Negative ion binding energies (BEs), in eV extracted from the TCSs for the actinide atoms Cf, Fm, and Md. GRS, MS- $n$ and EX- $n(n=1,2)$ represent respectively ground, metastable and excited anionic states. The experimental EAs, EXPT and the theoretical EAs (O’Malley \& Beck, 2009) and (Guo \& Whitehead, 1989) are also presented; N/A denotes not available. R-T Min is the energy position of the ground state R-T minimum in $\mathrm{eV}$. The ratio $\mathrm{R}$ is described in the text.

\begin{tabular}{|c|c|c|c|c|c|c|c|c|c|}
\hline Z & $\begin{array}{l}\text { Atom BEs } \\
\text { GRS }\end{array}$ & $\begin{array}{l}\text { EAs } \\
\text { EXPT }\end{array}$ & $\begin{array}{l}\text { BEs } \\
\text { MS-1 }\end{array}$ & $\begin{array}{l}\text { BEs } \\
\text { MS-2 }\end{array}$ & $\begin{array}{l}\text { BEs } \\
\text { EX-2 }\end{array}$ & $\begin{array}{l}\text { BEs } \\
\text { EX-1 }\end{array}$ & $\begin{array}{l}\text { BEs/EAs } \\
\text { Theory }\end{array}$ & $\begin{array}{l}\text { R-T Min } \\
\text { GRS }\end{array}$ & $\mathrm{R}$ \\
\hline 98 & Cf 3.32 & N/A & 1.70 & 0.955 & 0.577 & 0.272 & $\begin{array}{c}0.018[1] \\
0.010[1] \\
-0.777[2] \\
-1.013[2]\end{array}$ & 3.34 & 0.88 \\
\hline 100 & Fm3.47 & N/A & 1.79 & 1.02 & 0.623 & 0.268 & $\begin{array}{l}0.597[2] \\
0.354[2]\end{array}$ & 3.49 & 0.89 \\
\hline 101 & Md3.77 & N/A & 1.81 & 0.996 & 0.700 & 0.259 & $\begin{array}{l}1.224[2] \\
0.978[2]\end{array}$ & 3.78 & 0.90 \\
\hline
\end{tabular}

Sources: [1]. O’Malley and Beck, 2009; [2]. Guo and Whitehead, 1989

\section{Summary and Conclusion}

The low-energy electron elastic TCSs for the large actinide atoms Cf, Fm and Md calculated using the rigorous Regge pole method together with those of (Felfli \& Msezane, 2019; Msezane \& Felfli, 2021) now constitute the 
essential data for the actinide atoms. The extracted from the TCSs anion BEs have been compared with the existing theoretical EAs. Unfortunately, the theoretical EAs/BEs are riddled with uncertainties or are incomplete and the experimental EAs are unavailable. In particular, our BEs of the ground, metastable and excited anionic states of the formed negative ions during the collisions should be useful in the production of unambiguous and reliable BEs of the actinide negative ions.

We have discovered new manifestations in the low-energy electron scattering TCSs of the Cf, Fm and Md atoms; namely, fullerene molecular behavior near threshold. Also, a polarization-induced metastable cross section with a dominant SR appearing very close to threshold has been found in the Cf TCSs. We have attributed these peculiar tunable behaviors in the TCSs to size effects and orbital collapse impacting significantly the polarization interaction. The flip over of the near threshold R-T minimum from the Bk TCSs to a SR in the Cf TCSs provides a sensitive probe of the electronic structure of these atoms, thereby permitting the first ever use of the R-T minimum and the SR as novel confirmation of $\mathrm{Cf}$ as a transitional element in the actinide series.

In conclusion, our calculated and delineated ground, metastable and excited states anionic BEs of the $\mathrm{Cf}^{-}, \mathrm{Fm}^{-}$and $\mathrm{Md}^{-}$anions formed during the electron collisions can now be used in sophisticated theoretical methods such as the Coupled-Cluster, Dirac R-matrix and MCDHF to construct the appropriate wave functions and for fine-structure energy calculations. Also, these anionic BEs should be useful in guiding the determination of reliable EAs regardless of their definition. Indeed, the Regge pole approach requires no assistance whatsoever from either experiment or other theory to obtain rigorous and unambiguous negative ion BEs of complex multi-electron systems.

\section{Acknowledgements}

Research was supported by the U.S. DOE, Division of Chemical Sciences, Geosciences and Biosciences, Office of Basic Energy Sciences, Office of Energy Research. The computing facilities of National Energy Research Scientific Computing Center, also funded by U.S. DOE are greatly appreciated.

\section{Conflict of interests}

The authors declare that there is no conflict of interests regarding the publication of this paper.

\section{References}

Andersen, T., Haugen, H. K., \& Hotop, H. (1999). Binding Energies in Atomic Negative Ions: III. J. Phys. Chem. Ref. Data, 28(6), 1511. https://doi.org/10.1063/1.556047

Aubin, S., Myrskog, S., Extravour, M. H. T., LeBlanc, L. J., McKay, D., Stummer, A., \& Thywissen, J. H. (2006). Rapid sympathetic cooling to Fermi degeneracy on a chip. Nat. Phys. 2, 384. https://doi.org/10.1038/nphys309

Bahrim, C., \& Thumm, U. (2000). Low-lying 3Po and 3Se states of $\mathrm{Rb}^{-}, \mathrm{Cs}^{-}$, and $\mathrm{Fr}^{-}$. Phys. Rev. A., 61, 022722. https://doi.org/10.1103/PhysRevA.61.022722

Belov, S., Thylwe, K. E., Marletta, M., Msezane, A. Z., \& Naboko, S. N. (2010). On Regge pole trajectories for a rational function approximation of Thomas-Fermi potentials. J. Phys. A., 43, 365301. https://doi.org/10.1088/1751-8113/43/36/365301

Belov, S. M., Avdonina, N. B., Felfli, Z., Marletta, M., Msezane, A. Z., \& Naboko, S. N. (2004). Semiclassical approach to Regge poles trajectories calculations for nonsingular potentials: Thomas-Fermi type. J. Phys. A Math. Gen., 37, 6943. https://doi.org/10.1088/0305-4470/37/27/006

Bilodeau, B. C., Scheer, M. M., Haugen, H. K., \& Brooks, R. L. (1999). Near-threshold laser spectroscopy of iridium and platinum negative ions: Electron affinities and the threshold law. Phys. Rev. A., 61, 012505. https://doi.org/10.1103/PhysRevA.61.012505

Borschevsky, A., Eliav, E., Vilkas, M. J., Ishikawa, Y., \& Kaldor, U. (2007). Transition energies of atomic lawrencium. Eur. Phys. J. D., 45, 115. https://doi.org/10.1140/epjd/e2007-00130-9

Burke, P. G., \& Tate, C. (1969). A program for calculating regge trajectories in potential scattering Comp. Phys. Commun., 1, 97. https://doi.org/10.1016/0010-4655(69)90003-4

Ciborowski, S. M., Liu, G., Blankenhorn, M., Harris, R. M., Marshall, M. A., Zhu, Z., ... Peterson, K. A. (2021). The electron affinity of the uranium atom. J. Chem. Phys., 154, 224307. https://doi.org/10.1063/5.0046315

Connor, J. N. L. (1990). New theoretical methods for molecular collisions: The complex angular-momentum approach. J. Chem. Soc. Faraday Trans., 86, 1627. https://doi.org/10.1039/ft9908601627 
de Alfaro, V., \& Regge, T. (1995). Potential Scattering. Amsterdam: North-Holland.

Dolmatov, V. K., Amusia, M. Y., \& Chernysheva, L. V. (2017). Electron elastic scattering off A@C60: The role of atomic polarization under confinement. Phys. Rev. A., 95, 012709. https://doi.org/10.1103/PhysRevA.95.012709

Eliav, E., Kaldor, U., \& Ishikawa, Y. (1995). Transition energies of ytterbium, lutetium, and lawrencium by the relativistic coupled-cluster method. Phys. Rev. A., 52, 291. https://doi.org/10.1103/PhysRevA.52.291

Felfli, Z., \& Msezane, A. Z. (2018a). Simple method for determining fullerene negative ion formation. Euro Phys. J., D72, 78. https://doi.org/10.1140/epjd/e2018-80420-9

Felfli, Z., \& Msezane, A. Z. (2018b). Conundrum in Measured Electron Affinities of Complex Heavy Atoms. Journal of Atomic, Molecular, Condensate \& Nano Phys., $5(2)$, 1-8. https://doi.org/10.26713/jamcnp.v5i2.1093

Felfli, Z., \& Msezane, A. Z. (2019). Negative Ion Formation in Low-Energy Electron Collisions with the Actinide Atoms Th, Pa, U, Np and Pu. Appl. Phys. Research, 11, 52. https://doi.org/10.5539/apr.v11n1p52

Felfli, Z., \& Msezane, A. Z. (2020), Low-Energy Electron Elastic Total Cross Sections for Ho, Er, Tm, Yb, Lu, and Hf Atoms. Atoms, 8, 17. https://doi.org/10.3390/atoms8020017

Felfli, Z., Belov, S., Avdonina, N. B., Marletta, M., Msezane, A. Z., \& Naboko, S. N. (2004). In J. Govaerts, M. N. Hounkonnou, \& A. Z. Msezane (Eds.), Proceedings of the Third International Workshop on Contemporary Problems in Mathematical Physics (pp. 218-232). World Scientific, Singapore.

Felfli, Z., Msezane, A. Z., \& Sokolovski, D. (2009). Resonances in low-energy electron elastic cross sections for lanthanide atoms. Phys. Rev. A., 79, 012714. https://doi.org/10.1103/PhysRevA.79.012714

Frautschi, S. C. (1963). Regge Poles and S-matrix Theory. Chapter X, New York: Benjamin.

Gibson, D., Davies, B. J., \& Larson, D. J. (1993). The electron affinity of platinum. J. Chem. Phys., 98, 5104. https://doi.org/10.1063/1.464935

Guo, Y., \& Whitehead, M. A. (1989). Electron affinities of alkaline-earth and actinide elements calculated with the local-spin-density-functional theory. Phys. Rev. A., 40, 28. https://doi.org/10.1103/PhysRevA.40.28

Hiscox, A., Brown, B. M., \& Marletta, M. (2010). On the low energy behavior of Regge poles. J. Math. Phys., 51, 102104. https://doi.org/10.1063/1.3496811

Hotop, H., \& Lineberger, W. C. (2003). Dye-laser photodetachment studies of $\mathrm{Au}^{-}, \mathrm{Pt}^{-}, \mathrm{PtN}^{-}$, and $\mathrm{Ag}^{-}$. J. Chem. Phys., 58, 2379. https://doi.org/10.1063/1.1679515

Johnson, W. R., \& Guet, C. (1994). Elastic scattering of electrons from Xe, Cs+, and Ba2+. Phys. Rev. A., 49, 1041. https://doi.org/10.1103/PhysRevA.49.1041

Leimbach, D., Karls, J., Guo, Y., Eliav, E., et al.. (2020). The electron affinity of astatine. Nat. Commun, 11, 3824. https://doi.org/10.1038/s41467-020-17599-2

Li, J., Zhao, Z., Andersson, M., Zhang, X., \& Chen, C. (2012). Theoretical study for the electron affinities of negative ions with the MCDHF method. J. Phys. B., 45, 165004. https://doi.org/10.1088/0953-4075/45/16/165004

Macek, J. H., Krstic, P. S., \& Ovchinnikov, S. Y. (2004). Regge Oscillations in Integral Cross Sections for Proton Impact on Atomic Hydrogen. Phys. Rev. Lett., 93, 183202. https://doi.org/10.1103/PhysRevLett.93.183203

Msezane, A. Z., \& Felfli, Z. (2018a). New insights in low-energy electron-fullerene interactions. Chem. Phys., 503, 50. https://doi.org/10.1016/j.chemphys.2018.02.005

Msezane, A. Z., \& Felfli, Z. (2018b). Low-energy electron scattering from fullerenes and heavy complex atoms: Negative ions formation. Euro Phys. J., D72, 173. https://doi.org/10.1140/epjd/e2018-90121-0

Msezane, A. Z., \& Felfli, Z. (2021). Low-Energy Electron Elastic Collisions with Actinide Atoms Am, Cm, Bk, Es, No and Lr: Negative-ion Formation. Atoms, 9, 84. https://doi.org/10.3390/atoms9040084

Mulholland, H. P. (1928). An asymptotic expansion for $\Sigma(2 \mathrm{n}+1) \exp (\hat{\mathrm{a}} \sigma(\mathrm{n}+1 / 2) 2)$. Proc. Camb. Phil. Soc., 24, 280. https://doi.org/10.1017/S0305004100015796

O’Malley, S. M., \& Beck, D. R. (2009). Valence calculations of actinide anion binding energies: All bound 7p and 7s attachments. Phys. Rev. A., 80, 032514. https://doi.org/10.1103/PhysRevA.80.032514 
Omnes, R., \& Froissart, M. (1963). Mandelstam Theory and Regge Poles: An Introduction for Experimentalists, Chapter 2. New York: Benjamin.

Si, R., \& Froese Fischer, C. (2018). Electron affinities of At and its homologous elements Cl, Br, I. Phys. Rev. A., 98, 052504. https://doi.org/10.1103/PhysRevA.98.052504

Sokolovski, D., Felfli, Z., Ovchinnikov, S. Y., Macek, J. H., \& Msezane, A. Z. (2007b). Regge oscillations in electron-atom elastic cross sections. Phys. Rev. A., 76, 012705. https://doi.org/10.1103/PhysRevA.76.012705

Sokolovski, D., Msezane, A. Z., Felfli, Z., Ovchinnikov, S. Yu., \& Macek, J. H. (2007a). What can one do with Regge poles?. NIMB 261, 133. https://doi.org/10.1016/j.nimb.2007.04.057

Tang, R., Chen, X., Fu, X., Wang, H., \& Ning, C. (2018). Electron affinity of the hafnium atom. Phys. Rev. A., 98, 020501(R). https://doi.org/10.1103/PhysRevA.98.020501

Tang, R., Si, R., Fei, Z., Fu, X., Lu, Y., Brage, T., ... Ning, C. (2019). Candidate for Laser Cooling of a Negative Ion: High-Resolution Photoelectron Imaging of Th-. Phys. Rev. Lett., 123, 203002. https://doi.org/10.1103/PhysRevLett.123.203002

Thylwe, K. W. (2012). On relativistic shifts of negative-ion resonances. Eur. Phys. J. D, 66, 7. https://doi.org/10.1140/epjd/e2011-20530-4

Thylwe, K.-E., \& McCabe, P. (2014). Partial-wave analysis of particular peaks in total scattering cross sections caused by a single partial wave. Eur. Phys. J. D., 68, 323. https://doi.org/10.1140/epjd/e2014-50409-7

Zheng, W., Li, X., Eustis, S., Grubisic, A., \& Bowen, K. (2007). Anion photoelectron spectroscopy of Au-(H2O) 1, 2, Au2-(D2O) 1-4, and AuOH-. Chem. Phys. Lett., 444, 232. https://doi.org/10.1016/j.cplett.2007.07.036

\section{Copyrights}

Copyright for this article is retained by the author(s), with first publication rights granted to the journal.

This is an open-access article distributed under the terms and conditions of the Creative Commons Attribution license (http://creativecommons.org/licenses/by/4.0/). 